\title{
Field - Free and Field - Stimulated Electron Emission from Solids
}

\author{
Bernhard Gross \\ Instituto de Física de São Carlos - USP \\ Departamento de Física e Ciência dos Materiais \\ Grupo de Polímeros - Cx. Postal 369 \\ CEP 13560970 - São Carlos - SP \\ Carlos A.F. Pintão \\ Universidade Estadual Paulista - UNESP \\ Departamento de Física \\ Cx. Postal 473, Bauru - SP \\ Roberto Hessel \\ Universidade Estadual de São Paulo - UNESP \\ Departamento de Física \\ Cx. Postal 178, Rio Claro - SP \\ Received 2 March, 1998
}

\begin{abstract}
Electron irradiation of solids produces a backemission of secondary electrons (energies between 0 and $50 \mathrm{eV}$ ) and reflected primaries (energies between $50 \mathrm{eV}$ and that of the incident beam). For insulators, it is shown that an externally applied positive electric field penetrating into the solid material, energizes electrons generated by the primary irradiation and enables them to travel back to the surface of incidence and be emitted (stimulated secondary emission).
\end{abstract}

\section{Introduction}

Surfaces of solid materials irradiated by an electron beam become the source of electron emission in a direction opposite to that of the incoming beam. The energy of the emitted electrons extends from zero to the maximum of the irradiating beam. Electrons with energies between 0 and $50 \mathrm{eV}$ are classified as secondary electrons while those with higher energies are backscattered primaries. The application of an accelerating (positive) field enhances the emission of secondary electrons from the bulk of isolating materials. These additional electrons constitute a field - stimulated emission. Here we shall discuss the mechanism of the secondary emission and show some characteristic emission curves and energy distributions for conducting and for insulating materials. We emphasize the fact that the former do not exhibit field stimulated emission since the necessary electric field collapses in the material. For basic infor- mation on secondary emission we refer to the papers of Sternglass [1], Dekker [2], Seiler [3] and von Seggern [4].

\section{Measurement method}

Materials discussed here as representatives of the whole field are Teflon FEP and Platinum. Data on other materials are found in the Thesis of C.A.F.Pinto [5]. Targets were circular disks with an area of $7.1 \mathrm{~cm}^{2}$ and thickness of 25 to 40 micron for insulators; thickness of metals was higher. Insulating targets had a nonmetallized surface of incidence and a metallized backside resting on a metal disk used as the rear electrode.

In front of the sample surface, Fig.1, at a distance of 2 to $3 \mathrm{~mm}$, was mounted a stainless steel grid (transparency $80 \%$ ) and on top of the measuring arrangement, still within the vacuum, was mounted a metal plate with 2 circular openings for passage of the ini- 
cident beam, either for irradiating the sample or for entering a Faraday cup. The latter was covered by a steel grid of the same transparency as the one described above. The plate was biased by a positive voltage of 70 $\mathrm{V}$ to ground to remove the low energy secondaries generated by the incident beam on the plate itself and on the grid. The potential of $70 \mathrm{~V}$ is insufficient to remove backscattered high-energy electrons but these move oppositely to the primaries and thus do not interfere with the measurement. The samples were irradiated with a vertically incident scanned beam from an electron generator built by R. Hessel (6) in the São Carlos Institute. Irradiation was pulsed and each pulse gave a single measuring point.

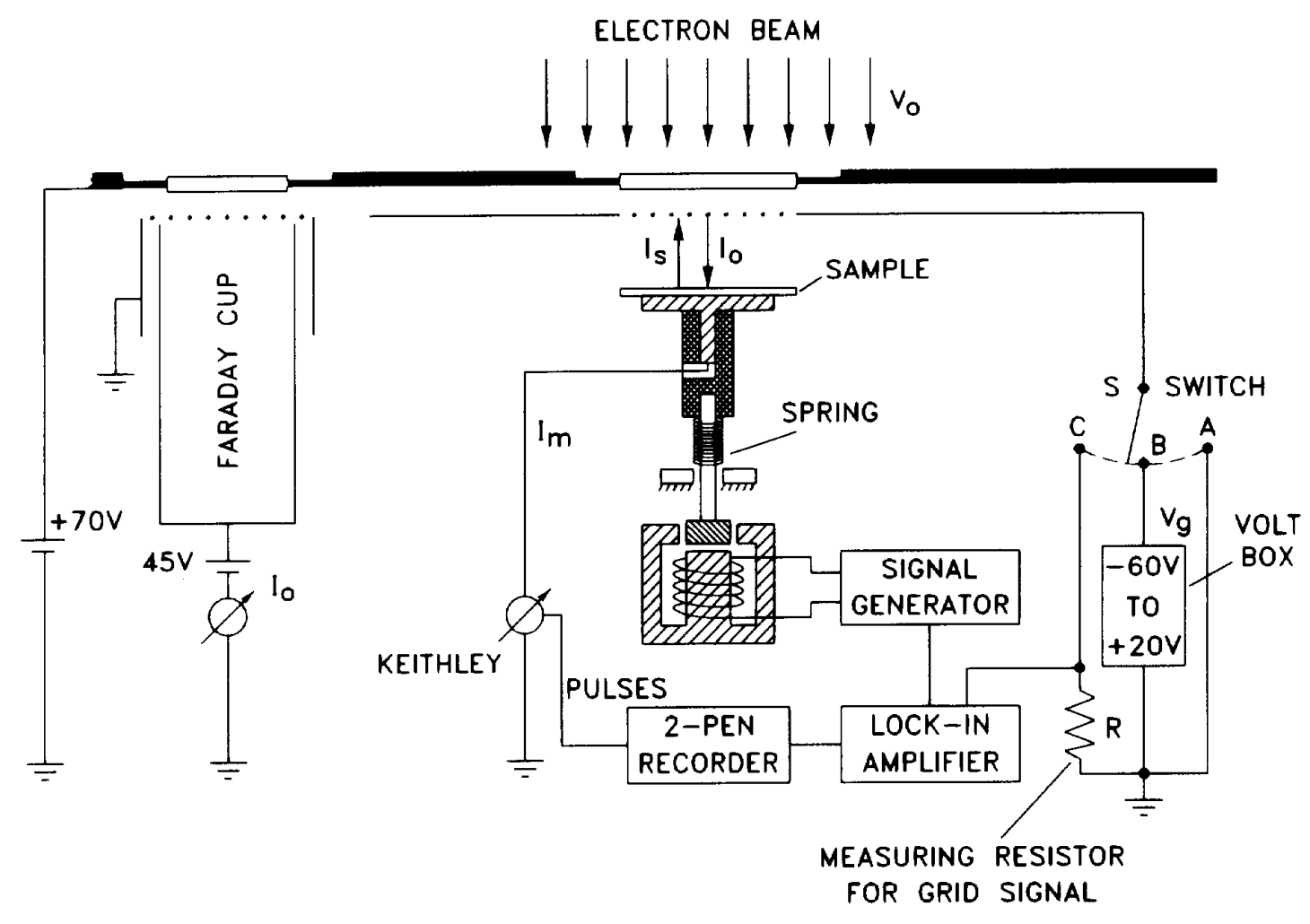

Figure 1. Measurement system.

The beam could be deflected from its normal position so as to incide on the Faraday cup which allowed a precise measurement of the beam current $I_{o}$. Vacuum was maintained within the whole system at a pressure of $10^{-6}$ Torr or less. The grid could be controlled by means of the switch $\mathrm{S}$. In position A the grid was grounded, in position $B$ it could be poled by means of a voltage box with potentials varying from $-60 \mathrm{~V}$ to +20 $\mathrm{V}$. In position $\mathrm{C}$ it was connected with the resistor $\mathrm{R}$ which provided the input for the lock-in amplifier mentioned below. The rear electrode of the sample was connected with the Keithley meter K1 which showed the measuring current $I_{m}$ and with the double pen recorder which registered the pulses of secondary electrons and of the vibrating capacitor (see below).

After each pulse a dielectric target usually carries a small positive or negative charge. To compensate these residual charges use was made of the characteristic emission curve of dielectrics. This curve exhibits two cross-over points with energies $\mathrm{E}_{\mathrm{I}}$ and $\mathrm{E}_{\mathrm{II}}$ where the amplitudes of the incident and the emission current are equal. Between $\mathrm{E}_{\mathrm{I}}$ and $\mathrm{E}_{\mathrm{II}}$ the emission current exceeds the incident current, while outside that interval the inverse prevails. A sample with a negative residual charge is irradiated with a beam of energy situated between $\mathrm{E}_{\mathrm{I}}$ and $\mathrm{E}_{\mathrm{II}}$. It loses negative charge because fewer electrons come in than go out. Eventually its potential approaches zero while the beam energy settles 
around $\mathrm{E}_{\mathrm{II}}$. Samples with a positive residual charge are irradiated with a beam of energy above $\mathrm{E}_{\text {II }}$ as discussed in [6].

After compensation, and before any new shot, the surface of the sample was examined whether it was indeed free of charge. The method of the vibrating capacitor was used [7]. The fixed plate was the grid which was connected to ground (position $\mathrm{C}$ of switch $\mathrm{S}$ ) over the measuring resistor $\mathrm{R}$. The other plate was the surface of incidence of the sample which was mounted on a steel rod which could move within a tubular guide and could be vibrated by a magnetic coil. If the surface of incidence of the sample was not fully discharged it would induce voltage oscillations on the fixed plate (the grid with the switch in position $C$ ) which eventually were indicated by the recorder. We believe we could maintain the surface of incidence in rest at a potential of at most $0.5 \mathrm{~V}$.

The current $I_{m}$ emerging from the rear of the sample was measured by a Keithley meter whose low exit was grounded. The same meter was connected over its rear contact with the recorder which registered the single pulses of the secondary emission. The use of the lock-in-amplifier prevented interference between the measurement of the secondary emission and the surface potential of the sample. The beam current $I_{0}$ was measured by the second Keithley connected to the Faraday cup to which the beam can be deviated. The pulses have a duration of less than half a second. After reaching a peak within a very short time, they are seen to decline, but for the amplitude measurements we took their peak values.

\section{Field - free and field - stim- ulated emission}

The grid was biased by a external voltage source by a voltage $V_{g}$ which could be varied from a negative minimum $V_{g}^{-}$to a positive maximum $V_{g}^{+}$. For $V_{g}^{-}<V_{g}<0$ one measures the secondaries which have enough energy to overcome the retarding field set up by $V_{g}$. Secondaries with energies betwen $V_{g}$ and 0 are turned back by the field of the grid and return to the surface of incidence. For $0_{+} \leq V_{g}<V_{g}^{+}$the field has inverted direction and becomes attractive. If the sample is a dielectric, the field extends into the bulk of the material, energizes secondaries generated by collisions with the penetrating beam, and enables them to travel back to the surface and be emitted. These electrons are the origin of the current component defined as field stimulated emission. We know that the intensity of the beam changes with depth in the dielectric but here we disregard this effect and use the box model, attributing to the incident primaries a constant range which depends only on their energy.

For metals no such field-stimulated emission exists since the field collapses within the material. So the section of the beam extending beyond the surface layer does not contribute to the emission, which remains constant.

\section{Effect of beam voltage}

The field-free emission for negative and zero voltage originates from collisions of the beam with near-surface atoms and is limited to a thin layer (the escape depth). With increasing positive voltage $V_{o}$ of an incident beam current of given intensity $I_{o}$, the depth of penetration increases (range increase) and the density of ionizationgenerated electrons along the range decreases. Therefore fewer electrons are available at the surface layer for emission which therefore decreases. Note that for each series of measurements intensity and voltage of the beam remained constant.

The energy spectrum of the field-free emitted electrons extends from zero to the beam energy. Here, following practice, we shall be concerned with secondary electrons, that is, those having energy no greater than $50 \mathrm{eV}$. Therefore, we define $V_{g}^{-}=-50 \mathrm{~V}$ as the maximum negative voltage to which our measurements shall extend; its maximum positive value is $V_{g}^{+}=+20 \mathrm{~V}$.

\section{Current equation}

The emission current $I_{s}$ is the difference between the measuring current $I_{m}$ and the value of the beam current $I_{o}$ defined as positive and constant throughout. Generally one has ([8], Eq. 1)

$$
I_{s}=I_{m}-I_{0}
$$

\section{Electron energy distribution function}

Let $g(V)$ be the number of secondary electrons of potential $V$ (or of energy eV). $g(V) \mathrm{dV}$ is their number between $V$ and $V+d V$. Multiplication with $e / s$, or charge 
emitted per second, gives the corresponding current element $(e / s) g(V) d(V)$. The expression for $I_{s}$ depends on whether the grid potential is negative or positive. In the first case one has

$$
I_{s}\left(V_{g}\right)=\int_{V_{g}^{-}}^{V_{g}}(e / s) g(V) d V, V_{g}^{-} \leq V_{g} \leq 0
$$

and in the second case

$$
I_{s}\left(V_{g}\right)=\int_{0^{+}}^{V_{g}}(e / s) g(V) d V, 0_{+} \leq V_{g} \leq V_{g}^{+}
$$

Eqs. (2) and (3) can be combined giving

$$
I_{s}\left(V_{g}\right)=\int_{V_{g}^{-}}^{V_{g}}(e / s) g(V) d V, V_{g}^{-} \leq V_{g} \leq V_{g}^{+}
$$

Normalizing this equation gives on the left side $I_{g}^{*}(s)$. The right hand term is divided by $\int_{V_{g}^{-}}^{V_{g}^{+}}(e / s) g(V) d V$.

Combining the normalized left-and right-side expressions gives

$$
I_{s}\left(V_{g}\right)=\int_{V_{g}^{-}}^{V_{g}} g^{*}(V) d V, V_{g}^{-} \leq V_{g} \leq V_{g}^{+}
$$

where $g^{*}$ is the normalized distribution function. Derivation and use of Eq. 1 give

$$
g^{*}\left(V_{g}\right)=\frac{d I_{m}\left(V_{g}\right) / d V_{g}}{I_{m}\left(V_{g}^{+}\right)-I_{0}}
$$

Nominator and denominator are negative, so $g^{*}(V)$ comes out positive.

\section{Experimental results}

Metal - The secondary emission current for Platinum (area $7.1 \mathrm{~cm}^{2}$ ) is shown in Fig. 2 for a beam current of $2 \times 10^{-8} \mathrm{~A}$ and voltages of 300 to $4000 \mathrm{~V}$. In all cases the currents become constant when the retarding voltage reaches zero and they remain so for increasing (positive) voltages $\left(0_{+}<V_{g}<20 \mathrm{~V}\right)$. Therefore there does not exist a stimulated emission from the metal. When the grid voltage becomes negative, the current emission falls fast and for $-14 \mathrm{~V}$ it has come down to $7 \times 10^{-9}$ A. It depends strongly on the beam voltage $V_{0}$, decreasing with increasing values of $V_{0}$. The emission current decreases by a factor of 1.7 for a variation of $V_{0}$ from $300 \mathrm{~V}$ to $4000 \mathrm{~V}$. As explained before the increasing beam range, at constant total beam current, decreases the specific ionization along its range and thus reduces the number of electrons available for emission at the surface.

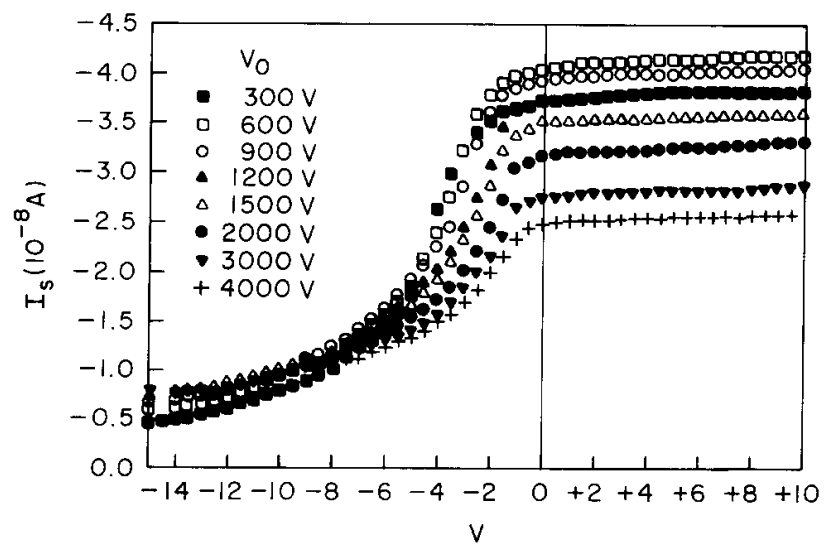

Figure 2. Secondary emission current from Platinum as a function of emission voltage, beam current $2 \times 10^{-8} \mathrm{~A}$, beam voltages from $300 \mathrm{~V}$ to $4000 \mathrm{~V}$.

The electron energy profile of Platinun for a beam voltage $V_{0}=300 \mathrm{~V}$ is shown in Fig. 3. The values to the left of $\mathrm{eV}=0$ correspond to collisions between the incident electrons and atoms of the surface layer of the metal. Since the electrons thus generated are freely emitted and are counted provided they overcome the grid, the measured values of $g$ or $g^{*}$ indicate directly the corresponding emission energies and thus give the true energy profile. The profile is strongly peaked with a half width of $2.5 \mathrm{eV}$, with the peak situated at $-4 \mathrm{~V}$, within the field-free emission. The small section of the curve extending beyond zero is probably due to lack of precision of the measurement and scattered high energy electrons interacting with the grid and chamber walls. The absence of field stimulated emissions is confirmed.

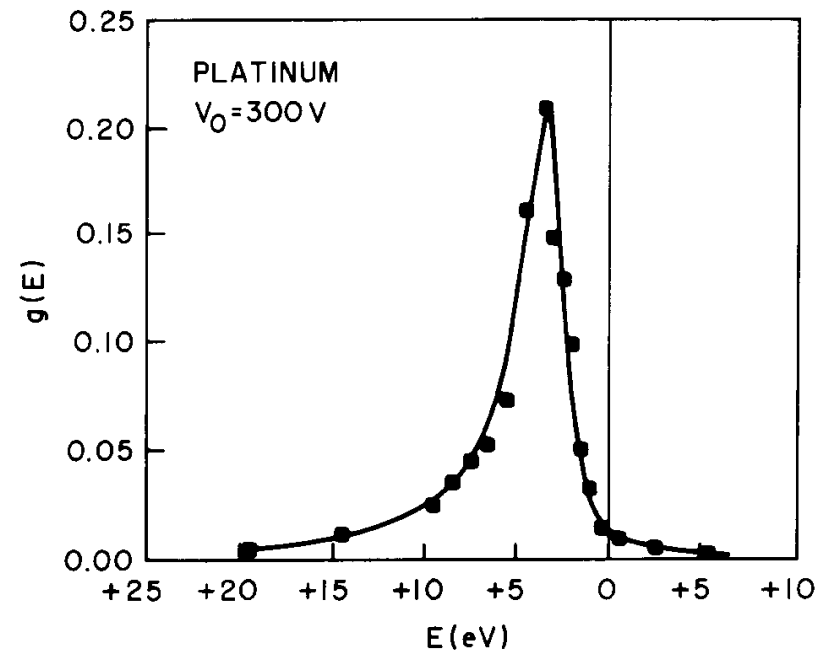

Figure 3. Electron energy profile for Platinum; beam current $2 \times 10-8 \mathrm{~A}$, beam voltage $300 \mathrm{~V}$. 
Insulator - The emission from the insulating polymer Teflon FEP (fluorethylene propylene) is shown in Fig. 4. The incident beam current in this case was 2.5 $\mathrm{nA}$ and the beam voltage varied from 200 to $2500 \mathrm{~V}$. The emission current does not become constant and independent on $V_{g}$ at $V_{g}=0$, but continues to increase straight on to the right of $\mathrm{eV}=0$ when one reaches positive energy values; since the field has changed direction and from retarding has become accelerating, additional electrons within the dielectric are returned to the surface and maintain the increase of $I_{s}$, this time due to the field-stimulated emission. The decrease of $I_{s}$ with increasing $V_{o}$ is explained as before by the decrease of specific ionization with increasing range.

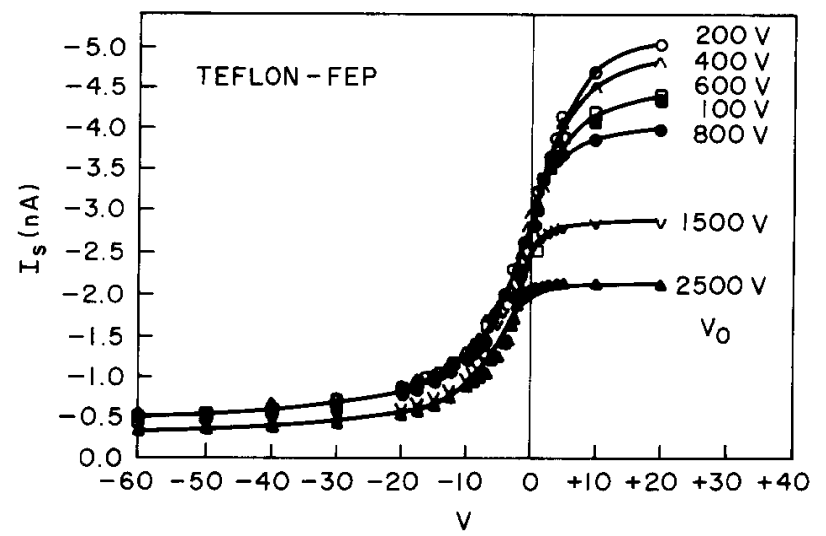

Figure 4. Secondary emission current from Teflon FEP as a function of emission voltage. Beam current $2.5 \mathrm{nA}$, beam voltages between 200 and $2500 \mathrm{~V}$.

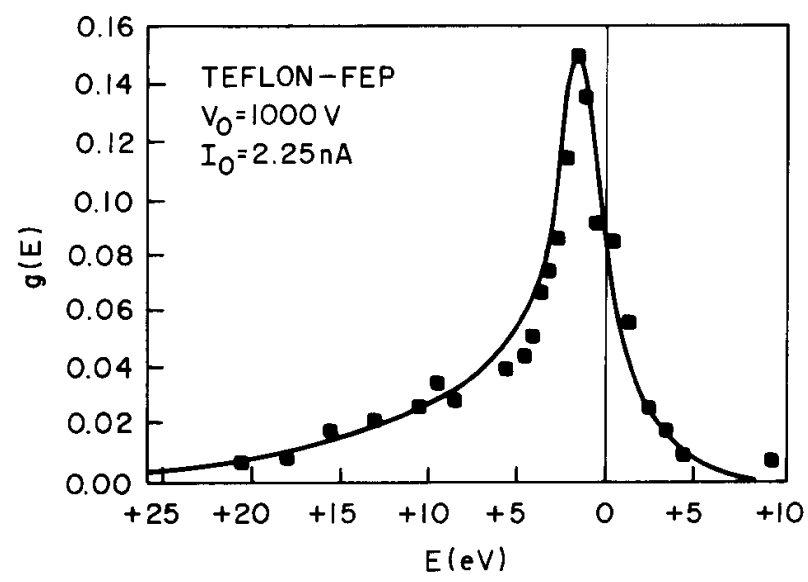

Figure 5. Electron energy profile for Teflon FEP. Beam current $2.5 \mathrm{nA}$, voltage $1000 \mathrm{~V}$.

The corresponding electron energy profile for $V_{0}=$ $1000 \mathrm{~V}$ is shown in Fig. 5.

The physical interpretation of the two sections of Fig. 5 for dielectrics differs. The left section, as discussed above, gives the true energy values of electrons originated by collisions with near-surface atoms. But the right side refers to collisions between beam electrons and atoms within the material, some distance from the surface. The electrons thus generated, move in the direction of the surface under the influence of the penetrating electric field but on the way lose some of their original energy due to conduction and collisions and when they arrive at the surface of emission, their energy differs from its original value. Therefore the computed value of $\mathrm{g}$ is slighty lower than the true value. The difference is believed to be small because the distance travelled by the electrons is short and there is indeed no lack of continuity of the curves of $I_{s}(g)$ versus $g$ where they cross the surface.

The profile, with a half-width of $4 \mathrm{eV}$, is much larger than for the metal.

The energy of the field-free emission decreases with decreasing distance from the emitting surface. However once one enters the region of stimulated emission the energy of the electrons begins to increase again due to the surging accelerating field. Therefore the energy scale in Fig. 5 decreases from an initial positive value to zero, but subsequently rises again to positive values.

In Figs. 3 and 5 , we have replaced $V_{g}$ by $\mathrm{eV}$, noting that our negative potentials as well as the positive ones correspond to positive kinetic energies.

A series of other measurements with various materials, including PETP and PVDF showed qualitatively the same behavior as described above [5].

\section{Conclusion}

The experimental results presented here give evidence that an externally applied positive electric field extending into a dielectric enhances the secondary electron emission by removing from the bulk of the material electrons generated there by the electron beam. Further experimental and theoretical developments will be needed for a satisfactory understanding and theory of the effect.

Recenty Dr. R. A. Frederickson [9] has investigated the total electron emission from insulating materials suggested for satellite shielding. He found a very strong influence of positive electric fields in the MV range, applied externally. He reported an incease of the total emission by factors up to 10 and exceeding by application of fields in the sample in the $10 \mathrm{KV} / \mathrm{cm}$ range. So 
he was the first to discover field stimulated emission for very high applied fields and beam energies.

\section{Acknowledgments}

One of the authors (B.G.) is greatly indebted to Dr. Frederickson (USAF, Phillips Lab. Hanscom AFB, MA 01731), now at Calif. Inst. Techn., Jet Propulsion Lab., Pasadena, Calif., for having called his attention to his various recent experiments on secondary emission from PMMA shielding material which proved the effect of stimulated emission.

All authors wish to extend their gratitude to Professor G.F. Leal Ferreira for his help in the completion and organization of the typescript and numerous discussions, and to the special editor of this issue of the Brazilian Journal of Physics, Prof. Dr. R. GerhardMulthaupt.

\section{References}

1. E. J. Sternglass, Phys. Rev. 95, 345 (1954).

2. A. Dekker, Secondary Emission. In: Solid Physics, Academic Press, New York 150, 251 (1958).

3. H. Seiler, J. Appl. Phys. 54, R.1 - R 18 (1983).

4. H. von Seggern, IEEE Trans. Nuclear Sci. 32 , $1503(1985)$

5. C.A.F. Pintão, PhD Thesis 1996, Institute of Physics of São Carlos, University of São Paulo, São Carlos.

6. R. Hessel, PhD Thesis 1990, Institute of Physics of São Carlos, University of São Paulo, São Carlos.

7. W. A. Zisman, Rev. Sci. Inst. 3, 361 (1932) L. Kelvin, Phil. Mag. 46, 82 (1898).

8. B. Gross and R. Hessel, IEEE Trans. El. Ins. 26, 18 (1991).

9. A. R. Frederickson and S. Woolf, J. Appl. Phys. 81, 834, (1997). 